\title{
Accelerated Aging in Ultrathin Films of a Molecular Glass Former
}

\author{
A. Sepúlveda, ${ }^{1}$ E. Leon-Gutierrez, ${ }^{1}$ M. Gonzalez-Silveira, ${ }^{1}$ C. Rodríguez-Tinoco, ${ }^{1}$ \\ M. T. Clavaguera-Mora, ${ }^{1}$ and J. Rodríguez-Viejo ${ }^{1,2, *}$ \\ ${ }^{1}$ Nanomaterials and Microsystems Group, Physics Department, Universitat Autònoma de Barcelona, 08193 Bellaterra, Spain \\ ${ }^{2}$ MATGAS Research Center, Campus UAB, 08193 Bellaterra, Spain
}

(Received 4 February 2011; revised manuscript received 2 May 2011; published 7 July 2011)

\begin{abstract}
We report the thermodynamic measurement of the enthalpy released during the aging of supported films of a molecular glass former, toluene, at temperatures well below the glass transition temperature. By using microfabricated devices with very short equilibration times (below $1 \mathrm{~s}$ ), we evidence a remarkable variation of the relaxation rate on decreasing film thickness from $100 \mathrm{~nm}$ down to a $7 \mathrm{~nm}$ thick film. Our results demonstrate that surface atoms are more efficient than bulk atoms in attaining low energy configurations within the potential energy landscape.
\end{abstract}

DOI: 10.1103/PhysRevLett.107.025901

PACS numbers: 65.60.+a, 64.70.pj

The creation of low-enthalpy glasses by quenching a supercooled liquid requires infinitely slow cooling rates that are unreachable over conventional laboratory time scales. It has been shown that vapor deposition at temperatures close to but below the glass transition temperature $T_{g}$ can produce glasses with enthalpies reduced by tens of $\mathrm{J} / \mathrm{g}$, relative to glasses obtained from a liquid [1-3]. In the case of indomethacin, samples cooled from the liquid would require thousands of years to reach identical values of the limiting fictive temperature obtained for films deposited from a vapor phase [2]. Other molecular glasses [3-8], also exhibit high stability after growth from the vapor at appropriate temperatures, so the phenomenon is observed in the general case.

Aging is the process by which a glass spontaneously transforms into a more stable glass as the result of molecular rearrangements occurring at temperatures below the glass transition temperature. This process is of extraordinary relevance in the field of polymers and glass materials, as the glasses may undergo changes in their physical properties and performance after a prolonged exposure to a given temperature.

Confinement has a pronounced influence on glass transition, and many studies have addressed the variation of $T_{g}$ with size ([9], and references therein). As a general rule, supported thin films composed of a variety of glasses show a depression in $T_{g}$ when their interaction with the substrate is weak, and an increase in $T_{g}$ when their interaction is strong. The existence of a gradient in the dynamics near a free surface was originally proposed by Keddie et al. for supported ultrathin polystyrene (PS) films, attributed to the presence of a liquidlike layer near the free surface [10]. Ellison and Torkelson [11] used fluorescence probes at various depths to demonstrate the gradient in $T_{g}$ through the film thickness. Although less studied, confinement has also been shown to have a significant influence on aging; this has been previously addressed by several authors [12-17], with most reporting a reduction in the physical aging rate near free surfaces. Priestley et al. [12] observed a factor of 2 decrease in the rate of structural relaxation at the surface of thin poly(methyl methacrylate) films. Pye et al. [15] found that the physical aging rate in $30 \mathrm{~nm}$ supported PS layers was reduced at all temperatures compared with thicker films. Kawana and Jones [17] found that PS films thicker than $18 \mathrm{~nm}$ aged below $T_{g}$, but $10 \mathrm{~nm}$ films did not; this suggests the presence of a surface layer with very fast dynamics for which aging completes before $10^{3} \mathrm{~s}$. Physical aging may also be influenced by the variation of $T_{g}$ in confined systems. Simon and co-workers [14] found that accelerated aging occurs in stacked PS thin film layers, in which the selected aging temperature was set constant with respect to $T_{g}$ for every thickness. Boucher et al. recently suggested that it may be possible to reconcile these contradictory results, by recording the relaxation data using a time scale shorter than the stabilization time to the desired aging temperature [18]. Recently a new device was reported with shorter thermal diffusion lengths which led to stabilization after a few seconds [19]. Our microfabricated calorimetric device $[20,21]$ has a time constant below $100 \mathrm{~ms}$ for cooling; therefore, the characteristic equilibration time at a given temperature after a thermal jump is roughly below $0.5 \mathrm{~s}$.

To our knowledge, no thermodynamic measurements have been made of the physical aging behavior of ultrathin films below $10 \mathrm{~nm}$, mainly due to the experimental difficulty of accessing thermodynamic parameters in such thin layers over very short times. Aging experiments performed with conventional setups typically require hundreds of seconds of equilibration time and may extend to weeks or more to obtain reliable data. The short equilibration times achieved in this work expand by more than two decades in time a conventional study on aging.

Here, we analyze the aging behavior of toluene thin films down to $7 \mathrm{~nm}$. We use the fictive temperature variation as the major parameter for quantifying the increased stability of the aged films. Our results demonstrate that 
aging is heavily influenced by the thickness of the layers. Ultrathin films aged at $90 \mathrm{~K}$ over a few hours reach fictive temperatures close in value to those of very stable vapordeposited films. In contrast, the aging of thicker films $(\sim 100 \mathrm{~nm})$ is ineffective for attaining a low configuration in the potential energy landscape compared to equivalent thick films obtained from vapor; approximately 10 years are needed to reach the fictive temperature of the vapordeposited films.

The experimental setup and deposition procedures have been previously described [22]. Additional details are included as Supplemental Material (SM) [23]. All vapordeposited films (AD films) ranging from 7 to $100 \mathrm{~nm}$ nominal thickness are grown at $90 \mathrm{~K}$, with a growth rate of $0.10 \pm 0.015 \mathrm{~nm} / \mathrm{s}$. After deposition, the sample is immediately fast scanned (at $\sim 3 \times 10^{4} \mathrm{~K} / \mathrm{s}$ ) to above $T_{g}$, as the first calorimetric scan. Subsequent passive cooling at $\sim 500 \mathrm{~K} / \mathrm{s}$ reverts the film to the glassy state (fast-cooled films, FC) at $90 \mathrm{~K}$. The next fast-heating upscan is representative of a glassy sample that is refrozen from a liquid. The glassy refrozen samples (FC) are aged over various times at $90 \mathrm{~K}$, far below $T_{g}\left(T_{g}=117 \mathrm{~K}\right)$, and the heat capacity is measured immediately after aging. The temperature jumps almost instantaneously to the aging temperature (within $1 \mathrm{~s}$ ), and the temperature fluctuations during aging are maintained below $100 \mathrm{mK}$. The ability to explore aging times spanning $0.5-10^{5} \mathrm{~s}$ enables reliable physical aging rates to be obtained for very thin films. The heat capacity is evaluated under the quasiadiabatic criterion, in which the differential measurement at very fast heating rates $\left(10^{4}-10^{5} \mathrm{~K} / \mathrm{s}\right)$ is nearly adiabatic. Under this condition, the heat capacity of the sample can be evaluated from the differential voltages between two calorimetric cells (sample and reference) during a temperature excursion $[24,25]$. In order to provide some insights into the morphology of the films we carry out specific vaporization experiments that consist of upscans of $\sim 10$ and $100 \mathrm{~nm}$ thick $\mathrm{AD}$ and $\mathrm{FC}$ layers at different heating rates up to high temperatures $(\sim 220 \mathrm{~K})$. Vaporization shows up as a strong endotherm in the heat capacity curves. Our Cp data are consistent with a zero-order kinetics vaporization behavior. Based on these results, and because of the very small residence time in the supercooled liquid region, we infer the films, even the thinnest ones, do not undergo measurable dewetting during the temperature scans. We also assume the films are continuous. We emphasize the interpretation of our results described below may not change appreciable even if the thinnest films lack continuity.

The fictive and onset temperatures for every thickness are determined by integration of the heat capacity curves and extrapolation of the supercooled liquid line to the glass region (see SM [23] and Refs. [22,26] for more details). All measurements have been reproduced between 5-15 times to ensure sufficient statistics, with error bars corresponding to the standard deviation.

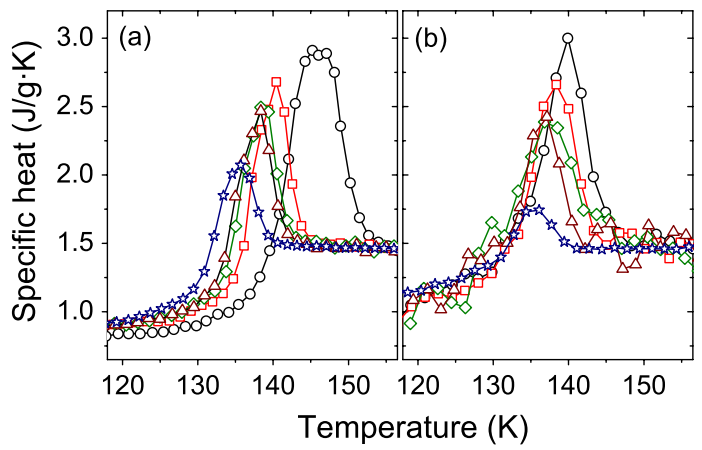

FIG. 1 (color online). Specific heat as a function of temperature for toluene thin films: (a) $100 \mathrm{~nm}$ thick, (b) $7 \mathrm{~nm}$ thick; for selected aging times at $90 \mathrm{~K}$. Blue star: fast cooled; brown uptriangle: $5 \mathrm{~min}$; green rhombus: $30 \mathrm{~min}$; red square: $720 \mathrm{~min}$; black circle: vapor deposited.

Figure 1 shows the specific heat for toluene films of various thicknesses after isothermal aging at $90 \mathrm{~K}$, provided for three selected times between $1 \mathrm{~s}$ and $720 \mathrm{~min}$. In $100 \mathrm{~nm}$ films, the aging results in a noticeable increase of the onset temperature and a larger overshoot. The thinner films behave differently; although the overshoot also increases with aging, the onset temperature changes only slightly.

The results obtained for the $\mathrm{AD}$ and $\mathrm{FC}$ samples are in agreement with a previous study [26] examining toluene films grown from vapor at around $\sim 0.8 T_{g}$. These previous results showed that the onset of the glass transition temperature $T_{\text {on }}$ and the limiting fictive temperature $T_{f}$ of the very stable glasses decreased with the film thickness. In contrast, the FC films cooled at $\sim 500 \mathrm{~K} / \mathrm{s}$ exhibit a filmthickness-independent glass transition temperature. These findings are consistent with observations in thin polymer films where cooling rates faster than $\sim 2 \mathrm{~K} / \mathrm{s}$ resulted in $T_{g}$ independent of thickness [14,27]. Figure 2 shows the variation with thickness of the fictive temperatures for toluene films aged at $90 \mathrm{~K}$ for times up to $30 \mathrm{~min}$. Only data for aging times of 5, 15, and 30 min are shown in the

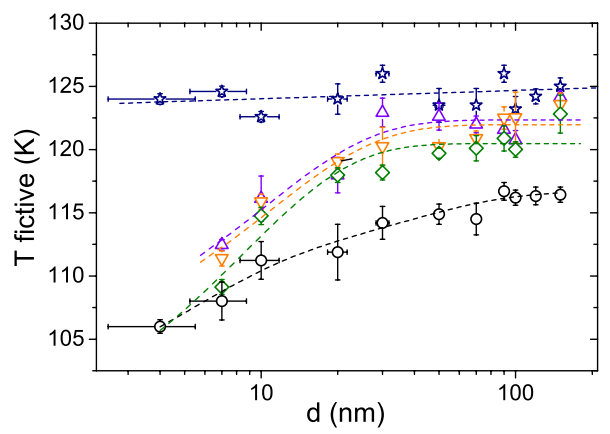

FIG. 2 (color online). Evolution of the fictive temperatures as a function of thickness for toluene films after various aging times at $90 \mathrm{~K}$. Blue star: fast cooled; violet up-triangle: 5 min; orange down triangle: $15 \mathrm{~min}$; green rhombus: 30 min; black circle: vapor deposited. The lines are provided only for visual guidance. 
figure. The complete set of data can be accessed as SM [23]. Figures 1 and 2 illustrate the general trend: during aging at $90 \mathrm{~K}$, the rate of increase in the thermodynamic stability of the films is thickness dependent. The main contribution of this work is the aging evolution of the thinnest films. Figure 2 shows there is a striking decrease of the fictive temperature during aging for the thinnest films in this study $(7 \mathrm{~nm})$. For the thickest films the evolution is markedly different; after prolonged aging up to $12 \mathrm{~h}$ (see SM [23]), the aged FC glass has increased its thermodynamic stability, but it is still far from the values reached by films grown from the vapor at the optimum temperature.

The analysis of aging at $90 \mathrm{~K}$ is focused on the values of the fictive temperature. $T_{f}$ reflects the structural stabilization of the film and is independent of the heating rate, quite important in view of the scan rates used in this study. Figure 3 shows the evolution of $T_{f}-T_{a}$ for $T_{a}=90 \mathrm{~K}$ as a function of aging time for films ranging in thickness from 7 to $100 \mathrm{~nm}$. The horizontal lines correspond to the fictive temperature for 7 and $100 \mathrm{~nm}$ thick vapor-deposited stable films. This figure illustrates the remarkable influence of the thickness on the aging properties of the glass. For a given aging time, there is a notable decrease in the fictive temperature as the film thickness decreases; that is, the aging dynamics accelerate with decreasing layer thickness. A bulk film (thickness $\geq 100 \mathrm{~nm}$ ) obtained from a liquid by a fast cooling procedure may need around 10 years $\left(2-3 \times 10^{8} \mathrm{~s}\right)$ to reach the low enthalpy of a vapordeposited glass. In contrast, a film with a thickness of $7 \mathrm{~nm}$ is able to reach a lower value of the fictive temperature (comparable to the highly stable vapor-deposited glass of the same thickness) in only $4 \mathrm{~h}\left(1-2 \times 10^{4} \mathrm{~s}\right)$. Our data demonstrate the ability of surface molecules to sample

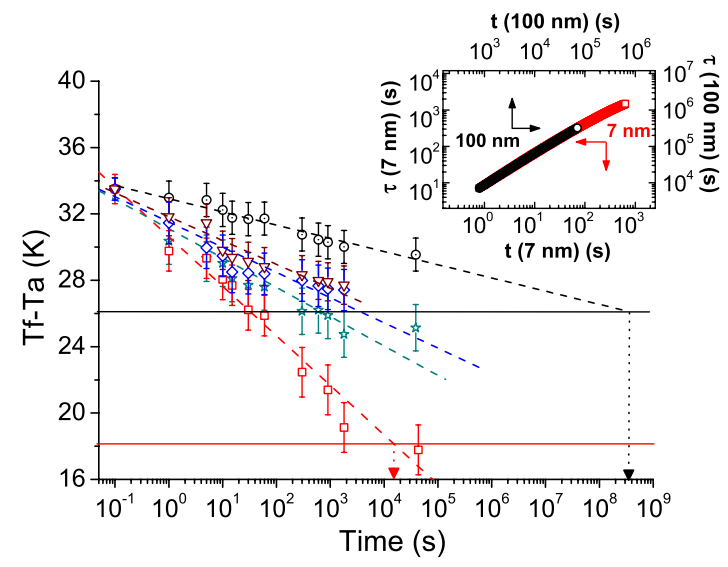

FIG. 3 (color online). $\quad T_{f^{-}} T_{a}$ evolution for $T_{a}=90 \mathrm{~K}$ as a function of aging time, for 7 (red square), 10 (green star), 20 (blue rhombus), 50 (brown down triangle), and 100 (black circle) $\mathrm{nm}$ thick toluene films. The inset shows the relaxation time vs time for the thinnest and thickest films obtained by fitting the experimental data with the model in the intermediate region. The arrows refer to the corresponding axis for each set of data. more efficiently the energy landscape attaining lower energy configurations than atoms in the interior of the films. This behavior supports very recent calculations in freestanding thin films by molecular dynamics simulation of a glass-forming binary Lennard-Jones mixture [28].

We further analyze our data using a simplified approach that neglects dynamic heterogeneities associated with a distribution of relaxation times [29]. However, (weak) nonexponential time evolution does occur due to the time-dependent relaxation time. The aging process corresponds to an evolution of $T_{f}\left(T_{\text {aging }}, t, d\right)$ for a film of thickness $d$ from its nonequilibrium $T_{f, 0, d}=T_{f}\left(T_{\text {aging, }}\right.$, $t=0, d)$ value to the smaller long-time equilibrium value, $T_{f}(t \rightarrow \infty, d)=T_{f, \mathrm{AD}, d}$. We use a first-order kinetic equation for the temporal evolution of $T_{f}$ with a time varying rate which is nonlinearly coupled with the mean relaxation time $[30,31]$. For each film thickness, the time dependence of the relaxation time $\tau\left(T_{f, t}\right)$ arises solely from the aging of $T_{f}(t)$

$$
\begin{gathered}
T_{f}=T_{f, \mathrm{AD}}+\left(T_{f, 0}-T_{f, \mathrm{AD}}\right) \exp \left[-\int d t^{\prime} / \tau\left(T_{f, t^{\prime}}\right)\right] \text { or } \\
d T_{f} / d(\ln t)=-\left[T_{f}(t)-T_{f, \mathrm{AD}}\right]\left[t / \tau\left(T_{f, t}\right)\right] .
\end{gathered}
$$

We use the intermediate domain of aging times where $d\left[d T_{f}(t) / d \ln (t)\right] / d t=0$ to evaluate $\tau\left(T_{f, t}\right)$ from Eq. (1). In this domain it has been shown [31] that

$$
d \ln \left(\tau_{r}\right) / \ln d t_{r}=1-\left(t_{r} / \tau_{r}\right),
$$

with $t_{r}=t / \tau\left(T_{f, 0, d}\right)$ and $\tau_{r}\left(T_{f, t, d}\right)=\tau\left(T_{f, t, d}\right) / \tau\left(T_{f, 0, d}\right)$. The inset of Fig. 3 shows a plot of the relaxation time vs time at the aging temperature of $90 \mathrm{~K}$ for the thinnest $\tau\left(T_{f, t, 7 \mathrm{~nm}}\right)$, and thickest $\tau\left(T_{f, t, 100 \mathrm{~nm}}\right)$ films. The red curve $(d=7 \mathrm{~nm})$ collapses with the black one $(d=100 \mathrm{~nm})$ when plotting it as $10^{-3} \tau\left(T_{f, t, 7 \mathrm{~nm}}\right)$ vs $10^{-3} t$. That is, the ratio of the average relaxation time $\tau\left(T_{f, 0}\right)$ between the 100 and $7 \mathrm{~nm}$ thick films is $\tau\left(T_{f, 0,100 \mathrm{~nm}}\right) / \tau\left(T_{f, 0,7 \mathrm{~nm}}\right) \sim 1000$. The change in the relaxation time is not related to any intrinsic variation of the glass transition temperature for the FC films, which at $t=0$ is thickness independent (see Fig. 2). These measurements also reveal the importance of using a very short equilibration time when dealing with ultrathin films; i.e., for a standard 5-10 min equilibration time at a given temperature, the fictive temperature of a $10 \mathrm{~nm}$ glassy film would have decreased by an impressive $10 \mathrm{~K}$.

The thickness dependence of the relaxation time helps to explain the mechanism of stabilization of vapor-deposited films. Our results support the interpretation of the molecular mechanisms underlying the enhanced stability of vapordeposited glasses which is based on the idea that, during deposition and before they are buried by new molecules impinging on the surface, surface molecules can sample more efficiently the potential energy landscape, reaching more stable configurations [2]. The relaxation time for 
these events is coupled to the structural configuration via the barrier height between neighboring basins of the energy landscape. The molecular rearrangements are affected by the free surface and the system has a higher probability to explore and attain lower configurational sites on the energy landscape which could be altered by the near surface. For bulk films, aging of glass obtained from a liquid is largely ineffective, since inner molecules are buried under other molecules and require extremely long times to reach more stable sites. However, for the thinnest films in this study $(7 \mathrm{~nm}), 50 \%$ of the total number of molecules in the film are located within 2-4 molecular diameters of the surface. In this environment, the mobility of the overall layer is largely enhanced and the system can reach more stable configurations with a higher efficiency. Although not explicitly included in the previous discussion we cannot rule out the influence of the substrate interface on the observed enhanced dynamics.

In summary, we observed superaccelerated aging in supported ultrathin glassy films of toluene. The thinnest films attained the low enthalpy of vapor-deposited layers within a few hours, whereas the thicker films would require tens of years to reach the same energy state as vapordeposited films. These results confirm the existence of surface domains having faster relaxation times that become dominant during the structural relaxation of the thinnest layers. Our data provide a strong test to theories and simulations on glass dynamics relative to the strong decrease of the relaxation rate in near-surface regions and the ability of surface molecules to reach deeper states within the potential energy landscape.

This work is supported by MICINN MAT2007-61521, MAT2010-15202, and by the Generalitat de Catalunya through SGR2009-01225. A. S. thanks CONACYT for support.

*Corresponding author. javier.rodriguez@uab.es

[1] S. F. Swallen, K. L. Kearns, M. K. Mapes, Y. S. Kim, R. J. McMahon, J. Robert, M. D. Ediger, T. L. Yu, and S. Satija, Science 315, 353 (2007).

[2] K. L. Kearns, S. F. Swallen, M. D. Ediger, W. Tu, and L. Yu, J. Chem. Phys. 127, 154702 (2007).

[3] K. L. Kearns, S. F. Swallen, M. D. Ediger, T. Wu, Y. Sun, and L. Yu, J. Phys. Chem. B 112, 4934 (2008).

[4] E. Leon-Gutierrez, A. Sepulveda, G. Garcia, M. T. Clavaguera-Mora, and J. Rodriguez-Viejo, Phys. Chem. Chem. Phys. 12, 14693 (2010).

[5] E. Leon-Gutierrez, G. Garcia, M. T. Clavaguera-Mora, and J. Rodríguez-Viejo, Thermochim. Acta 492, 51 (2009).

[6] K. Ishii, H. Nakayama, S. Hirabayashi, and R. Moriyama, Chem. Phys. Lett. 459, 109 (2008).
[7] K. Ishii, H. Nakayama, R. Moriyama, and Y. Yokoyama, Bull. Chem. Soc. Jpn. 82, 1240 (2009).

[8] L. Zhu and L. Yu, Chem. Phys. Lett. 499, 62 (2010).

[9] M. Alcoutlabi and G. B. McKenna, J. Phys. Condens. Matter 17, R461 (2005).

[10] J. L. Keddie, R. A. Jones, and R. A. Cory, Europhys. Lett. 27, 59 (1994).

[11] C. J. Ellison and J. M. Torkelson, Nature Mater. 2, 695 (2003).

[12] R.D. Priestley, C. J. Ellison, L. J. Broadbelt, and J.M. Torkelson, Science 309, 456 (2005).

[13] R. D. Priestley, Soft Matter 5, 919 (2009).

[14] Y.P. Koh and S. L. Simon, J. Polym. Sci. B 46, 2741 (2008).

[15] J.E. Pye, K. A. Rohald, E. A. Baker, and C. B. Roth, Macromolecules 43, 8296 (2010).

[16] B. W. Rowe, B. D. Freeman, and D. R. Paul, Polymer 50, 5565 (2009)

[17] S. Kawana and R. A. L. Jones, Eur. Phys. J. E 10, 223 (2003).

[18] V. M. Boucher, D. Cangialosi, A. Alegría, J. Colmenero, J. Gonzalez-Irun, and L. M. Liz-Marzan, Soft Matter 6, 3306 (2010).

[19] T. Hecksher, N. B. Olsen, K. Niss, and J. C. Dyre, J. Chem. Phys. 133, 174514 (2010).

[20] A. F. Lopeandia, J. Rodriguez-Viejo, M. Chacón, M. T. Clavaguera-Mora, and F. J. Muñoz, J. Micromech. Microeng. 16, 965 (2006).

[21] A. F. Lopeandia, J. Valenzuela, and J. Rodriguez-Viejo, Sens. Actuators A 143, 256 (2008).

[22] E. Leon-Gutierrez, G. Garcia, A.F. Lopeandia, J. Fraxedas, M. T. Clavaguera-Mora, and J. RodriguezViejo, J. Chem. Phys. 129, 181101 (2008).

[23] See supplemental material at http://link.aps.org/ supplemental/10.1103/PhysRevLett.107.025901 for more details of the experimental setup and the analysis of the fictive temperature. The file also contains more data of heat capacity for various thicknesses and Tonset and Tfictive for various aging treatments.

[24] M. Y. Efremov, F. Schiettekatte, M. Zhang, E. A. Olson, A. T. Kwan, R. S. Berry, and L. H. Allen, Phys. Rev. Lett. 85, 3560 (2000).

[25] M. Y. Efremov, E. A. Olson, M. Zhang, S. L. Lai, F. Schiettekatte, Z.S. Zhang, and L.H. Allen, Thermochim. Acta 412, 13 (2004).

[26] E. Leon-Gutierrez, G. Garcia, A. F. Lopeandia, M. T. Clavaguera-Mora, and J. Rodriguez-Viejo, J. Phys. Chem. Lett. 1, 341 (2010).

[27] Z. Fakhraai and J. A. Forrest, Phys. Rev. Lett. 95, 025701 (2005).

[28] Z. Shi, P. G. Debenedetti, and F. H. Stillinger, J. Chem. Phys. 134, 114524 (2011).

[29] M. D. Ediger, Annu. Rev. Phys. Chem. 51, 99 (2000).

[30] R. Richert, Phys. Rev. Lett. 104, 085702 (2010).

[31] K. Chen and K. S. Schweizer, Phys. Rev. E 78, 031802 (2008). 